\title{
Permanent Pacemaker Implantation Challenged by an Elevated Left Hemidiaphragm
}

Farla Jean-Louis ${ }^{1 *}$ and Asma Syed ${ }^{1,2}$

${ }^{1}$ Brookdale Hospital and Medical Center, Brooklyn, New York, USA.

${ }^{2}$ Brookdale Hospital and Medical Center, SUNY Downstate Medical Center, Brooklyn, New York, USA.

*Corresponding Author: Farla Jean-Louis, Brookdale Hospital, Brooklyn, New York, USA.

Received date: December 02, 2020; Accepted date: December 15, 2020; Published date: February 20,2021

Citation: Jean-Louis, F., Syed A., (2021) Permanent Pacemaker Implantation Challenged by an Elevated Left Hemidiaphragm Clinical Medical Reviews and Reports 3(1); DOI:10.31579/2690-8794/055

Copyright: (C) 2021 Farla Jean-Louis, This is an open access article distributed under the Creative Commons Attribution License, which permits unrestricted use, distribution, and reproduction in any medium, provided the original work is properly cited.

\section{Abstract}

This is a case of a 93 year old patient who presented with a syncopal episode due to infra-nodal atrio-ventricular block (AVB) which required placement of a permanent pacemaker (PPM). The initial pre-procedure chest X-ray (CXR) showed an elevated left hemidiaphragm with shifting of the heart to the right making PPM implant challenging.

Keywords: cardiac conduction; computed tomography; challenging pacemaker; elevated hemidiaphragm; chestX-Ray; CXR; PPM

\section{Introduction}

Cardiac conduction devices were first created in the 1950s and now, it is estimated that 1.25 million patients have PPM placed each year worldwide $[1,2]$. It is important to be knowledgeable about the variant anatomy that may affect lead placement prior to the procedure. To evaluate for common post procedural complications such as pneumothorax, perforation of the heart muscle or a vein or presence of an air pocket, a CXR is crucial. [3]. Current literature most commonly describes incorrect positioning of the leads due to its migration, retraction or misplacement.

There is no literature describing an abnormal position of a PPM due to an elevated left hemidiaphragm. Here, we present a 93 year old woman who presented with a syncopal episode due to an infra-nodal AVB for whom implantation of a PPM was challenged by a marked elevation of her left hemidiaphragm.

\section{Case Presentation}

This is a 93 year old woman with a past medical history of diabetes type 2 and hypertension who presented after a recurrent episode of syncope. She reported dizziness prior to the episode. On admission, her vital signs were normal with a blood pressure of $135 / 98 \mathrm{~mm} \mathrm{Hg}$ and a heart rate of 67 beats per minute. The computed tomography of the head without contrast was negative. The CXR on 1/11/2020 showed a marked elevation of the left hemidiaphragm (Figure 1). Her laboratory tests demonstrated a normal complete blood count, blood urea nitrogen, creatinine and troponin. Her urine drug toxicology screening was negative and her echocardiogram showed normal systolic and diastolic function, an elevated pulmonary artery pressure of $35-40 \mathrm{~mm} \mathrm{Hg}$ (normal $=25 \mathrm{~mm} \mathrm{Hg}$ ) and absence of any valvular disease. The holter showed several episodes of second degree AVB and evidence of infra-nodal AVB for which a pacemaker implant was recommended.

During the surgical procedure, due to her elevated left hemi-diaphragm and shifting of structures from left to right, the ventricular (RV) lead was difficult to place and took an unusual turn to be positioned in the right ventricular apex. The sensing, impedance and threshold numbers were noted to be within normal limits despite the unusual fluoroscopic position. The post procedural CXR on 1/16/2020 demonstrated the unusual position of the right ventricular lead (Image 2). The patient tolerated the procedure well and was discharged. Since implant she has had multiple office visits and pacemaker checks which have all demonstrated normal PPM function.

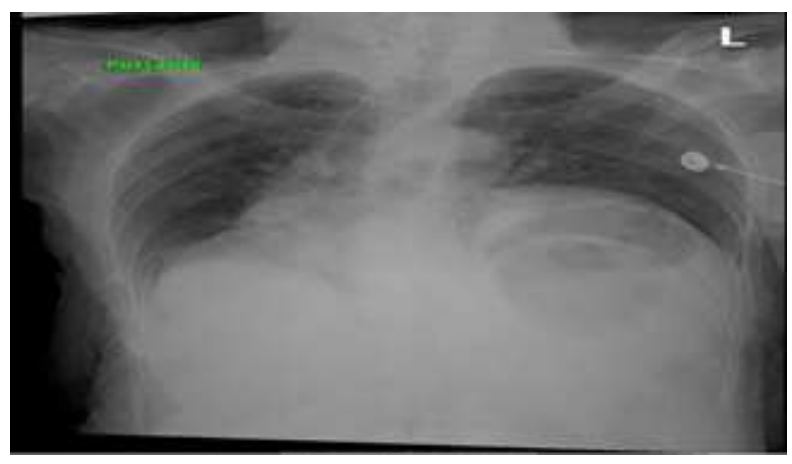




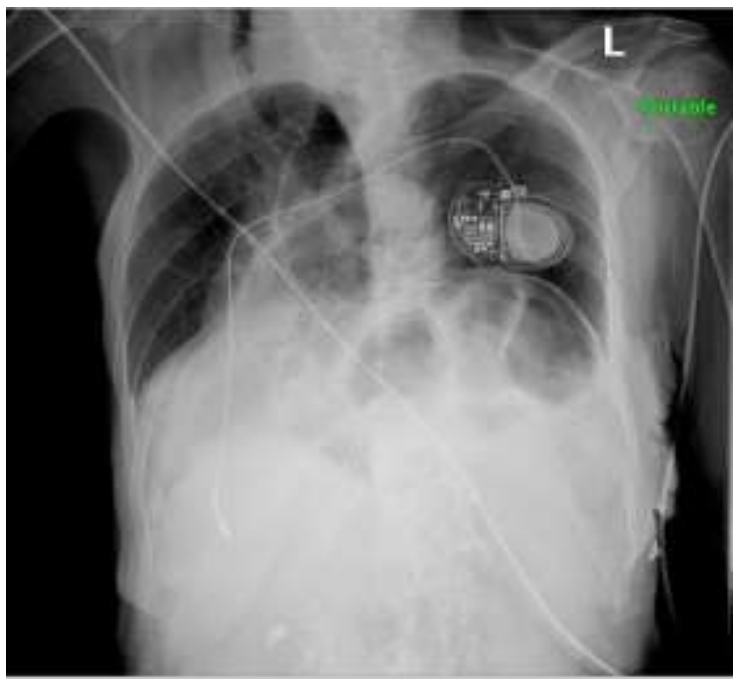

Figure 2. CXR on $1 / 16 / 2020$ shows a marked elevation of the left hemidiaphragm with the PPM in place.

\section{Discussion}

Cardiac conduction devices, such as PPM, have been improving the mortality and morbidity of patients with symptomatic bradycardia and AVB for several years. In fact, June 2010 officially marked the 50th anniversary of the first successful human cardiac PPM implantation [4].

Radiology plays an important role in the pre-procedural planning, placement and continued long term follow up. A post procedural CXR helps detect any micro or macro dislodgement when compared with baseline imaging along with any post procedural complications including pneumothorax or hematoma. Our patient was found to have an incidental finding of a marked elevation of the left hemidiaphragm. This anatomical variant rendered the PPM placement more challenging.

Current literature describes other cases of anatomical variants which necessitated unusual placement of the wires. For example, entering the left ventricle because of dextro transposition of the great arteries after a Senning/Mustard repair or having to pace the right atrium/ventricle from a persistent left sided superior vena cava and the coronary sinus [5]. There were no other similar case reports or research data describing a patient with an elevated left hemidiaphragm leading to a challenging PPM implantation.

\section{Conclusion}

Prior to PPM implantation, radiological studies are crucial in evaluating for an anatomical variant to be better prepared for the procedure. Here we presented an interesting case of unusual position of the RV lead fluoroscopically due to elevated left hemidiaphragm and shifting of structures to the right with normal pacemaker and lead function.

\section{References}

1. Torres-Ayala, S. C., Santacana-Laffitte, G., \& Maldonado, J. (2014). Radiography of Cardiac Conduction Devices: A Pictorial Review of Pacemakers and Implantable Cardioverter Defibrillators. Journal of Clinical Imaging Science, 4, 74.

2. Carrión-Camacho, M. a . R., Marín-León, I., Molina-Doñoro, J. M., \& González-López, J.R. (2019). Safety of Permanent Pacemaker Implantation: A Prospective Study. Journal of Clinical Medicine, 8(1), 35.

3. Aguilera, A. L., Volokhina, Y. V., \& Fisher, K. L. (2011). Radiography of Cardiac Conduction Devices: A Comprehensive Review. RadioGraphics, 31(6), 1669-1682.

4. Beck, H., Boden, W. E., Patibandla, S., Kireyev, D., Gupta, V., Campagna, F., Cain, M. E., \& Marine, J. E. (2010). 50th Anniversary of the First Successful Permanent Pacemaker Implantation in the United States: Historical Review and Future Directions. The American Journal of Cardiology, 106(6), 810 818.

5. Dipoce, J., Bernheim, A., \& Spindola-Franco, H. (2015). Radiology of cardiac devices and their complications. The British Journal of Radiology, 88(1046), 20140540. 